\title{
INTRODUCTION
}

The present volume represents an attempt to compare the industrial relations systems of the United States and Japan through an institutional-behavioral approach. Significant emphasis is placed both upon the institutional aspects of each system and upon empirical data concerning perception of industrial workers at the plant level in both countries. This balanced emphasis arises from our belief that an industrial relations system is an integral part of the socioeconomic structure of society and, even more specifically, that worker perceptions play an extremely important role in the eventuation of any system of industrial relations.

In this brief introduction, we wish to present some of the more important assumptions that have guided our quest for understanding of all industrial workers and the systems of industrial relations within which they must live and work. There are certain assumptions on which we are quite confident of our agreement. As partners in a joint project, we have necessarily shared certain common frames of reference in our research venture. On the other hand, as social scientists from different cultural backgrounds, we have frequently encountered and struggled with dissimilarities. By definition, a research program involving multiple cultures must reject a single framework based solely upon the experience accumulated within one of the societies involved. Unless some degree of detachment exists between the research framework and all the cultures under investigation, a study claiming to be cross-cultural will result merely in the imposition of ethnocentrism. In this research program involving two cultures, we have tried to recognize the need for a third, a rather neutral, frame of reference which will be useful in exploring worker perceptions of industrial relations in the United States and Japan. Throughout the volume, a consistent effort has been made to maintain this sort of cross-cultural framework. 
Industrial relations is primarily an applied field in which the parties involved-management, workers, and government-seek solutions to practical problems. The logic of each interest group toward others is problem-centered and action-oriented. In this volume, we shall attempt to provide practitioners of industrial relations in each society with clues-as suggested by the analysis-regarding the nature of the more pressing problems and their possible solutions. Efforts will be made to reconcile a cross-cultural approach with the action-oriented viewpoint.

In this introduction, four objectives are pursued. First, we shall seek to develop briefly a concept of industrial relations system which will be useful throughout the volume. Our discussion here will be limited in length, since the chapter that follows deals more rigorously with this subject. Second, we shall develop the concept of equilibrium and change in industrial relations. Relevant questions dealt with include the manner in which patterns of industrial relations develop and survive in a culture, and the means by which continual changes are brought about in such patterns. Third, we shall seek to clarify the focus of the present study on the basis of the foregoing concepts. In so doing, an explanation will be offered as to how a study such as ours can be of specific value in the industrial development of a society. Finally, a brief preview of the volume will be given to aid the reader in understanding the over-all design of the study as the various chapters unfold.

\section{Industrial Relations System}

In this study, the term "industrial relations system" refers basically to the integral pattern of mutual accommodation among three "actors," namely, workers, management, and government, in an industrial society. However, we are principally concerned with the pattern of relationships in industrial organizations between the first two actor groups-workers and management. We employ the term "system" because industrial relations represents a relatively independent, tightly knit set of interactions among individuals and groups, the principal components of the "system," whether at the plant, enterprise, industry, or national level.

A system of industrial relations is created primarily through industrialization, and is a function of (1) the inherent logic of industrialization (industrial demands) and (2) the relevant traditional and contemporary context of the given culture (cultural demands). The effect of inherent industrial demands upon indus- 
trial relations systems is in many respects universal, continuous, and accumulative. Some industrial demands may be manifested in such specific goals as productivity, loyalty, diligence, and creativity of workers in industrial organizations. Others, however, may be better expressed in relative terms. For example, a proper balance may be sought between excessive mobility and stability in employment, co-operation and competition among workers, centralization and decentralization of authority, and specialization and generalization in division of work.

Industrial demands may or may not be entirely compatible with the cultural demands, or contextual forces that affect a system of industrial relations. Some cultural demands may be supportive of, while others may be in direct conflict with (or at least irrelevant to), inherent industrial demands. It should be recognized, of course, that in an industrial society, cultural demands are themselves subject to constant change as a result of the industrialization process. The result of this reciprocal action between the imperatives of culture and industrialization is a constant renewing of the context of industrial relations in each society.

Within this context of mutual interaction and constant change, therefore, the pattern of the industrial relations system in a given society at any given time represents the net result of the perception, evaluation, and mutual accommodation of workers, management, and government. Assuming that these principal parties are composed of intelligent and able individuals striving for the best accommodative balance possible, the industrial relations system prevailing in a society will represent the most rational pattern obtainable under prevailing circumstances in the sense that ends are pursued which are viewed as possible within the conditions of the situation. Such rationality in industrial relations, naturally, is not always realized at the enterprise, industry, or national level. It should be pointed out, however, that when a number of nations are compared, variations among industrial relations systems do not necessarily imply "failure" of any one system in the sense of deviation from a given, or "best," model. The effectiveness of a particular industrial relations system lies more in the degree of achieved accommodation compatible with the economic and social goals of the society than in conformity with prevailing patterns in the most industrially advanced nations. Thus the patterns of industrial relations prevailing in different nations may vary substantially and yet be equally effective and appropriate in the respective situations. 


\section{Equilibrium and Changes in Industrial Relations}

Viewed as a system, then, industrial relations represents a relatively stable pattern of interactions among participating groups. But, like all aspects of social relations, it is also a dynamic process, in the sense that the pattern is subject to constant forces for readjustment. A cross-sectional analysis tends to overemphasize the "system" aspects of industrial relations while neglecting the "process" aspects. This point deserves particular attention in cross-cultural analyses, which often lack sufficient time perspective to evaluate properly the various forces in society which shape and reshape the patterns of industrial relations.

Under normal circumstances, a fair degree of equilibrium exists in any system of industrial relations, representing the conditions of balance previously described. This equilibrium reflects, for example, the balance of power relationships among the parties involved, whose respective positions are in turn subject to the logic of industrialization as well as cultural demands. When a pattern is structured, it tends to persist and maintain its stability through selfperpetuation. But, from time to time, some contingency is likely to develop and create certain tensions which, in turn, will threaten the existing state of equilibrium.

Many illustrations could be cited of such events leading to disequilibrium. For example, principal actor groups may change in organization, membership, size, ideological orientation, or strength. Whatever the cause, the resulting gain or decline in the power of one group will create disequilibrium and affect the whole configuration of industrial relations. However, except in times of emergency, such as war, revolution, and economic crisis, the shift in role and status of parties in industrial relations is apt to be a gradual one.

New patterns of interactions are more likely to emerge in response to an accumulation of more predictable evolutionary pressures. For example, a shift in the political power structure of society may alter the balance among groups. Or new labor, material, and product market conditions may exert pressures upon management to modify the way in which it has dealt with workers. Changes in education and family systems, in living standards and other social conditions also create disequilibrium in industrial relations, though their impact usually is less dramatic and abrupt than the impact of political or economic changes. Since various shifts in context seem destined to occur on a continuous basis, some degree of disequilibrium in industrial relations is likely to be 
the rule. In short, there are likely to be continual pressures to which the principal parties must adjust in their mutual accommodation process.

Both as individuals and institutions, the three actors in industrial relations must perceive and appraise their relative positions and the state of their environment continuously, so that each may intelligently redefine its attitudes and policies in a manner which will tend to restore equilibrium and order in the industrial relations system. Failure to do so will further magnify the forces of disequilibrium, and is likely to result in serious industrial conflict, social unrest, and perhaps even complete collapse of an economy. In some extreme situations, drastic and costly action, such as a revolution or a coup, too often appears to be the only effective solution.

\section{Focus of the Present Study}

The present cross-cultural study deals with industrial relations both as a system and as a process. From the first point of view, we regard the present state of industrial relations in each culture as a self-sufficient system tending toward equilibrium. We have found many differences between the United States and Japan, and many similarities. These we have tried to analyze in a manner that will result in a better understanding of each system through the mirror provided by the other.

In this respect, the worker perception data we have gathered through a questionnaire survey bear particular significance. Since industrial workers represent an important actor group, their perceptions comprise a significant source of pressure in any industrial relations system. As such, worker perceptions can provide certain insights in an area thus far dealt with primarily by social scientists with a more institutional orientation. We make no claim that such perceptual data are the most important or the most reliable source of insight concerning industrial relations problems. Neither do we wish to underestimate the role that various institutional disciplines play. On the contrary, we believe that true understanding in this complex phase of human affairs necessitates integration of knowledge from various disciplines. We realize that such a task is both difficult and risky at best. The chapters which follow will allow the reader to appraise whatever success we have achieved in this integration of perceptual data with findings of other scientific disciplines.

Analysis of the "process" aspect of industrial relations in each 
society is especially helpful in dealing with two basic aspects of the study. First, such analysis can provide some answers as to why, in each culture, we find the particular pattern of industrial relations which does exist. Obviously, the historical material found in Chapters 3 and 4 , though necessarily brief, represents our major effort in this direction. Even such a limited treatment of the evolutionary process should prove to be helpful in providing an indispensable time perspective in analysis.

Second, the "process" approach is especially significant in identifying and evaluating underlying symptoms of disequilibrium in a prevailing system of industrial relations. Since disequilibrium is the central cause of future changes, identification of such symptoms is of paramount importance to the principal actors in an industrial society. Findings of this study suggest three different sources of pressure toward disequilibrium, which, in turn, is likely to result in tensions and remedial changes. Though merely mentioned here, they will be discussed at much greater length in Parts II and III as they apply to the systems of industrial relations in the United States and Japan.

1. Gaps in perceptions among different worker groups concerning those industrial relations practices which they consider most desirable may be a source of considerable tension, and hence may be indicative of future change. Such perceptual differences, reflecting differing interests and value orientation, may cause conflicting patterns of worker behavior among groups. These behavior conflicts are a potential source of friction and disequilibrium in the industrial relations system.

2. Differences in worker perceptions and management practices in industrial relations may also cause pressure for changes in the system of industrial relations. Such differences may be reconciled either by changes in worker perceptions or by modifications in management practices. When the environmental context supports worker perceptions, it is more likely that changes will have to take place in management practices. If, on the other hand, practices are more in line with contextual demands, then worker perceptions will be more likely to adapt in order to restore equilibrium.

3. Even when perception-practice differences do not exist, gaps may be noted between contextual forces and perceptionpractice patterns. Even though no obvious signs of disequilibrium may be observed in worker-management relations, underlying environmental forces can often exert even more pressure than internal conditions upon industrial relations. Therefore, such gaps fre- 
quently are useful indicators of changes which may take place in an industrial relations system.

It is not our intention to set up here ideal models of present or future industrial relations systems. The systems found in the United States and Japan each have unique features, with each possessing some potential for change. Nevertheless, utilizing the limited data currently available, we have been able to identify certain sources of potential disequilibrium in each nation. From this standpoint, the volume should prove of interest to practitioners as well as scholars in the field.

\section{A Preview}

The first chapter in this volume discusses the process of industrialization and its impact on the growth of industrial relations systems. Major functional areas of industrial relations are also discussed as they relate to the analysis in Part II. Some typical assumptions are examined concerning how a pattern of industrial relations emerges and is related to the cultural demands in society.

Chapter 2 primarily covers the framework and methodology of the present study. The rationale for our focus upon worker perceptions is followed by a discussion of methods employed in data collection in the United States and Japan.

Chapters 3 and 4 are devoted to the historical development of industrial relations in the United States and Japan, respectively. Political, economic, social, and cultural backgrounds, and their relevance to change in industrial relations patterns, are discussed.

Part II, which includes Chapters 5 to 11 , examines specific aspects of industrial relations in the United States and Japan. Included are such functional areas as motivation to work, employment commitment, supervisors and men, status systems, remuneration and rewards, personnel decisions, and labor unions. For each area, the discussion is developed according to the following outline:

a) a theoretical analysis of the specific topic;

$b$ ) present practices, and the environmental context of industrial relations in the United States and Japan;

c) worker perceptions of industrial relations related to the practice and context of each society;

d) summary.

The final chapter presents a summary, or profile, of worker perceptions concerning the industrial relations system in each of 


\section{xviii INTRODUCTION}

the two countries studied. An attempt is made here to develop a theory which may account for the observed differences in the "system" aspects of industrial relations in the two societies. Finally, from a "process" point of view, each system is examined with respect to sources of disequilibrium and the nature of possible change. 